\title{
PRESYNAPTIC CALCIUM DIFFUSION AND THE TIME COURSES OF TRANSMITTER RELEASE AND SYNAPTIC FACILITATION AT THE SQUID GIANT SYNAPSE ${ }^{1}$
}

\author{
ROBERT S. ZUCKER*,2 AND NORMAN STOCKBRIDGE $\ddagger^{*} 3$ \\ *Department of Physiology-Anatomy, University of California, Berkeley, California 94720 and $\ddagger$ Department of Physiology, \\ Duke University, Durham, North Carolina 27710
}

Received May 26, 1982; Revised September 20, 1982; Accepted January 17, 1983

\begin{abstract}
At the squid giant synapse, a presynaptic action potential is accompanied by an influx of calcium ions that continue to be detectable with arsenazo III microspectrophotometry for several seconds. Nevertheless, transmilter release occurs phasically, lasting only aboul 2 msec. If a second action potential follows within about $100 \mathrm{msec}$ after the first, it releases more transmitter. In this paper, we present a mathematical model of intracellular calcium diffusion with binding to fixed cytoplasmic sites, active extrusion at the surface, and influx during an action potential, to predict the distribution of intracellular calcium following an action potential. With a square law relation between submembrane calcium and transmitter release, the model predicts the phasic release of transmitter and the magnitude and time course of synaptic facilitation following an action potential, as well as the relatively long persistence of free intracellular calcium.
\end{abstract}

A presynaptic action potential has at least two effects on the release of transmitter at nerve terminals: (1) Shortly after the action potential invades the terminal, the rate of transmitter release rises rapidly to a peak for $1 \mathrm{msec}$, then drops back to very low levels in another few milliseconds. (2) The release process is facilitated by the action potential, such that a second action potential arriving after the first will release more transmitter. This synaptic facilitation decays with several components having time constants ranging from a few milliseconds to a few minutes in various preparations. Recent experiments on the squid giant synapse confirm that the presynaptic influx of calcium through voltage-dependent channels is responsible both for the phasic release of transmitter following a spike (Llinás et al., 1981a, b) and for the facilitation of subsequent spike-evoked release (Charlton et al., 1982). Measurements of changes in intracellular calcium in the presynaptic terminal using the calcium indicator arsenazo III show that the average free calcium concentration rises about $50 \mathrm{nM}$ during the falling phase of the action potential, but persists in the terminal for a long time, disappearing roughly exponentially with a time

\footnotetext{
${ }^{1}$ This work was supported by National Institutes of Health Grants NS 15114 and NS 11613.

${ }^{2}$ To whom correspondence should be addressed at Department of Physiology-Anatomy, University of California, Berkeley, CA 94720.

${ }^{3}$ Present address: Department of Physiology, New York Medical College, Valhalla, NY 10595.
}

constant of a few seconds (Miledi and Parker, 1981; Charlton et al., 1982). By considering the diffusional movement of presynaptic calcium, we have now succeeded in doing two things: (1) we can account for the rapid decline of phasic transmitter release following a spike, and for the slower decline in facilitation, with a single process having to do with submembrane presynaptic calcium, and (2) we can reconcile these calciumdependent processes occurring in the 1 - to $100-\mathrm{msec}$ range in squid giant synapses with the seconds-long decline in average presynaptic free calcium concentration.

We present here a simple mathematical model of the movements of calcium ions in presynaptic terminals to account for spike-evoked phasic transmitter release, synaptic facilitation, and the measured post-spike changes in presynaptic free calcium. The presynaptic terminal is represented as a long cylindrical process, $50 \mu \mathrm{m}$ in diameter, bounded by a membrane through which calcium ions enter uniformly from the external medium during an action potential and then diffuse inward. Transmitter release is influenced only by the calcium immediately beneath (within $10 \mathrm{~nm}$ of) the membrane, where fusion of transmitter-containing vesicles with release sites occurs. Calcium entering the terminal is immediately apportioned between a fraction remaining free and a fraction bound to fixed cytoplasmic sites. Calcium is removed from the terminal by a surface membrane calcium transport system, and a resting inward calcium current is 
included to yield a non-zero calcium level in steady state. The components of this model are outlined in Figure 1.

\section{Materials and Methods}

Several processes interact to determine the temporal and spatial distribution of calcium ions in the presynaptic terminal. Each process may be represented by a simple equation, and the result of their interaction may be predicted by the simultaneous solution of the equations. We begin with the diffusion equation in cylindrical coordinates with radial symmetry, assuming no longitudinal or angular gradients:

$$
\frac{\partial C a(r, l)}{\partial t}=\frac{D}{(\beta+1) r} \frac{\partial}{\partial r}\left(r \frac{\partial C u(r, l)}{\partial r}\right)
$$

where $C a(r, t)$ is the intracellular free calcium concentration at distance $r$ from the central axis of the presynaptic terminal and at time $t, D$ is the diffusion constant of calcium in aqueous solution $\left(6 \times 10^{-6} \mathrm{~cm}^{2} / \mathrm{sec}\right.$; Hodgkin and Keynes (1957)), and $\beta$ is the ratio of bound calcium to free calcium in cytoplasm.

This equation is appropriate only for rapid binding of calcium to uniformly distributed, immobile, and nonsaturable binding sites in cytoplasm. These assumptions are undoubtedly simplifications, but they are not likely to be too far off. Tillotson and Gorman (1980) found that cytoplasmic calcium binding was high near the surface of molluscan cell bodies and lower in the center. These cells are nearly filled with a large nucleus; thus their low central binding is probably a property of the nucleus. The squid presynaptic terminal contains no nuclei. Nevertheless, some radial nonuniformities in calcium binding are likely to exist, due in part to a nonuniform distribution of intracellular organelles. The neurosecretory machinery, localized at the surface, is also likely to influence free calcium concentration. Thus $\beta$ should be a function of $r$, but no information on this functional form is available.

The assumption of instantaneous binding is also certainly a simplification. Nevertheless, equation 1 is appropriate as long as the calcium binding kinetics are rapid compared to the times required for diffusional equilibration. This is likely to be true, because otherwise the intracellular calcium concentration signal would show a spike followed by a plateau in response to a brief influx of calcium (Blaustein and Hodgkin, 1969). No such transient reflecting the dynamic binding of calcium is evident in arsenazo III absorbance records of presynaptic calcium concentration changes in an action potential (Charlton et al., 1982).

It is also not likely that calcium-binding sites are all totally immobile. Only sites on the plasma membrane would be absolutely fixed, but calcium-binding proteins and organelles are quite bulky and are not likely to move nearly as fast as free calcium ions; thus this approximation seems fairly reasonable.

Finally, calcium is bound to cytoplasm by a number of mechanisms, each of which is saturable (Brinley et al., 1977, 1978; Baker and Schlaepfer, 1978; Blaustein et al., 1978; Brinley, 1978). However, the wide range of affinities of different binding systems, with dissociation constants from $<1$ to $>10 \mu \mathrm{M}$, produces a system not likely to approach saturation following one or a few action potentials. We have chosen a value of $\beta=40$ as typical of values reported in the literature on axoplasmic calcium binding.

In our model we remove calcium from cytoplasm with a first-order plasma membrane extrusion pump, in accord with several studies of calcium efflux (Blaustein, 1976; DiPolo, 1976, 1979; Requena and Mullins, 1979). Although these studies differ in their interpretation of the mechanism of calcium extrusion, they are in general agreement on the kinetics of the pump, and we used a median value of $0.2 \mathrm{pM} / \mathrm{cm}^{2} \cdot \mathrm{sec}$ of calcium removed $/ \mu \mathrm{M}$ calcium concentration, corresponding to a pump rate $(P)$ of $2 \times 10^{-4} \mathrm{~cm} / \mathrm{sec}$. We used a nonsaturable pump because the extrusion process is reported to be nearly linear to above $1 \mu \mathrm{M}$ free internal calcium (Requena and Mullins, 1979). In our simulations (see below), the submembrane calcium never exceeds this order of magnitude of calcium concentration.

Free cytoplasmic calcium has been reported to be maintained at levels as low as $10 \mathrm{nM}$ in squid axoplasm (DiPolo et al., 1976); therefore, we chose this as the resting level of cytoplasmic calcium. This requires an inward resting calcium leak of $2 \mathrm{fM} / \mathrm{cm}^{2} \cdot \mathrm{sec}$ to balance

\section{CALCIUM DIFFUSION MODEL}

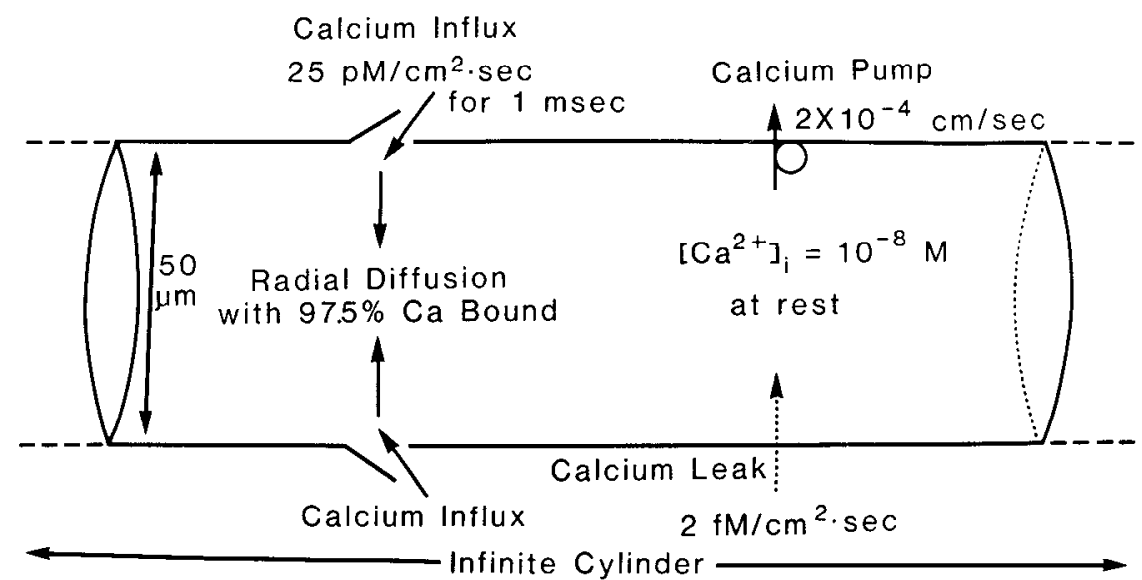

Figure 1. Schematic representation of the calcium diffusion model described in the text. 
the resting level of active extrusion at $10 \mathrm{~nm}$ free calcium

The influx of calcium ions during an action potential $\left(J_{\text {in }}\right)$ was approximated by fitting a rectangular pulse to the wavcform of calcium current during an action potential, as calculated by Llinás et al. (1981a). We used a 1msec influx of $25 \mathrm{pM} / \mathrm{cm}^{2} \cdot \mathrm{sec}$.

Equation 1 was solved with the influx and efflux of calcium at the surface as boundary conditions. A finite difference approximation for partial difference equations (Crank and Nicolson, 1947) was used to transform equation 1. The time domain was divided into intervals, $\Delta t$, and the terminal was divided into concentric annular tubular shells of thickness $\Delta r$. Then equation 1 becomes

$$
\begin{aligned}
& C a(r, t)=\frac{D \Delta t}{(\beta+1) r(\Delta r)^{2}}[(r+\Delta r / 2) C a(r+\Delta r, t-\Delta t)+(r \\
& -\Delta r / 2) C a(r-\Delta r, t-\Delta t)-2 r C a(r, t-\Delta t)]+C a(r, t-\Delta t)
\end{aligned}
$$

for each shell centered on $r$.

In order for this finite difference approximation to converge to the exact solution, sufficiently small time and distance intervals must be used; smaller values are needed when the temporal and spatial gradients are sharp. We used shell thicknesses of $10 \mathrm{~nm}$ for the outer 300 shells; i.e., down to $3 \mu \mathrm{m}$ below the membrane. Pilot simulations showed that we could treat the remaining axoplasmic core as one sink in the form of a rod of radius $2 r_{1}$ without appreciably affecting the results. We used 10$\mu \mathrm{sec}$ time intervals during action potentials and for 20 msec after them, $100-\mu \mathrm{sec}$ intervals until $200 \mathrm{msec}$ after a spike, 1-msec intervals until 2 sec after a spike, and finally 10-msec intervals for up to $20 \mathrm{sec}$ after a spike.

The innermost shell is centered at $r=r_{1}$, has thickness $2 r_{1}$, and flux only at its outer surface at $2 r_{1}$, so it may be represented by

$$
\begin{aligned}
C a\left(r_{1}, t\right)=\frac{D \Delta t}{2(\beta+1) r_{1}{ }^{2}}\left[C a\left(r_{1}+\Delta r, t-\Delta t\right)\right. \\
\\
\left.-C a\left(r_{1}, t-\Delta t\right)\right]+C a\left(r_{1}, t-\Delta t\right)
\end{aligned}
$$

where $r_{1}+\Delta r$ refers to the next shell surrounding the central rod. The outermost shell at radius $r_{0}$ is bounded by the plasma membrane and is influenced by calcium influx through voltage-dependent channels and the surface pump as well as diffusion into the interior. These processes are represented by the equation

$$
\begin{aligned}
C a\left(r_{0}, t\right)= & \frac{D \Delta t}{(\beta+1) r_{0}(\Delta r)^{2}}\left[( r _ { 0 } - \Delta r / 2 ) \left(C a\left(r_{0}-\Delta r, t-\Delta t\right)\right.\right. \\
& \left.\left.-C a\left(r_{0}, t-\Delta t\right)\right)\right]+\frac{J_{\text {in }} \Delta t}{\Delta r}+\left(1-P \frac{\Delta t}{\Delta r}\right) C a\left(r_{0}, t-\Delta t\right)
\end{aligned}
$$

The full system of equations 2 to 4 was solved on a digital computer.

\section{Results}

Figures 2 to 4 show the behavior of this model in response to one or two action potentials. In Figure $2 A$, the calcium concentration was integrated throughout the volume of the terminal to yield an average cytoplasmic concentration as a function of time during and after a single action potential. This should correspond to the arsenazo measurement of intracellular calcium. Due to signal-to-noise limitations, it has not been possible to record accurately the slow decline in arsenazo absorbance following a single action potential. However, our calculated average calcium concentration time course is similar to the decline in arsenazo absorbance following a brief tetanus, reproduced in Figure $2 A$ (Miledi and Parker, 1981; Charlton et al., 1982). We also note that the predicted increment in average internal free calcium is 17 $\mathrm{nM}$, which is consistent with the experimental order of magnitude estimate of $50 \mathrm{~nm}$ following a single action potential (Charlton et al., 1982).

However, the submembrane calcium which controls transmitter release behaves quite differently from the integral of total cytoplasmic calcium. The calcium entering at the membrane surface diffuses rapidly into the interior of the terminal, so that the initially sharp gradient of calcium at the surface is almost entirely dissi-
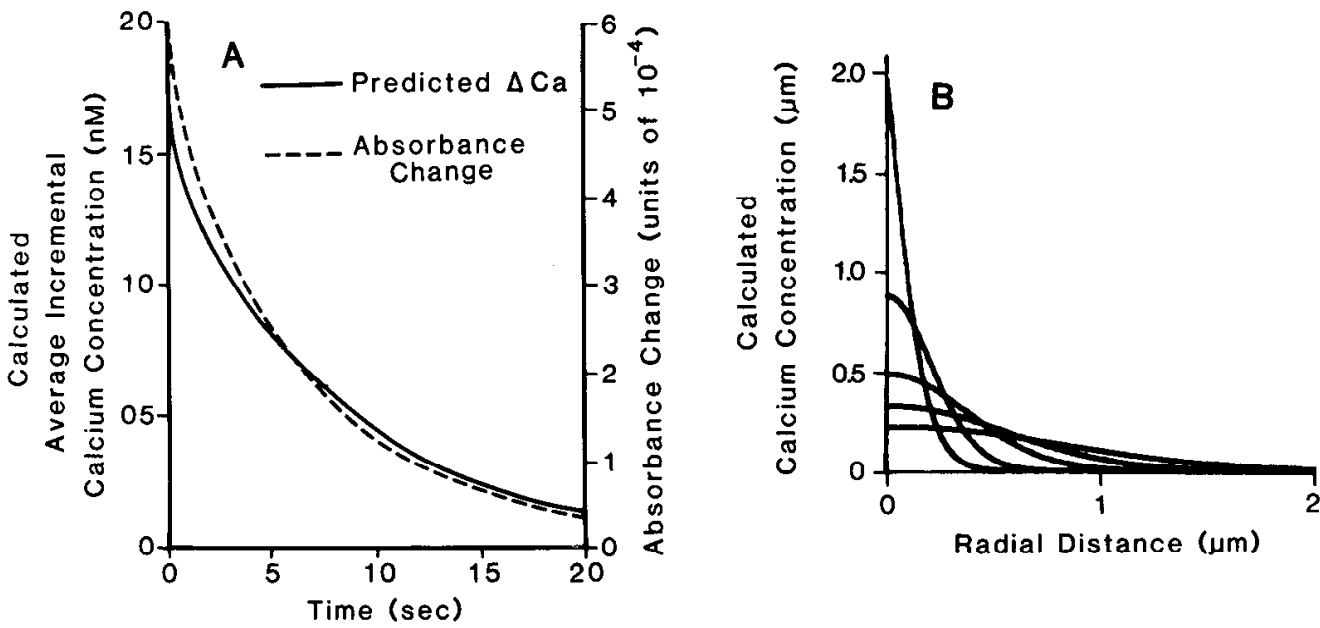

Figure 2. Predictions of a diffusion model of calcium movements in the presynaptic terminal and comparison to experimental results. $A$, Solid line: calculated average increase in calcium concentration following an action potential. The resting calcium level has been subtracted. Dashed line: measured post-tetanic change in differential absorbance at the wavelength pair $660-690$ nm, indicating the change in presynaptic free calcium concentration, in a terminal injected with arse $7 a z o$ III, at $15^{\circ} \mathrm{C}$; adapted from Charlton et al. (1982). Time is measured from the end of the action potential (solid line) or the end of a 2 -sec tetanus at $33 \mathrm{~Hz}$ (dashed line). B, Calculated presynaptic calcium concentration versus distance from the membrane at (from the top) 0 , $1,4,9$, and $19 \mathrm{msec}$ after the end of the action potential. 


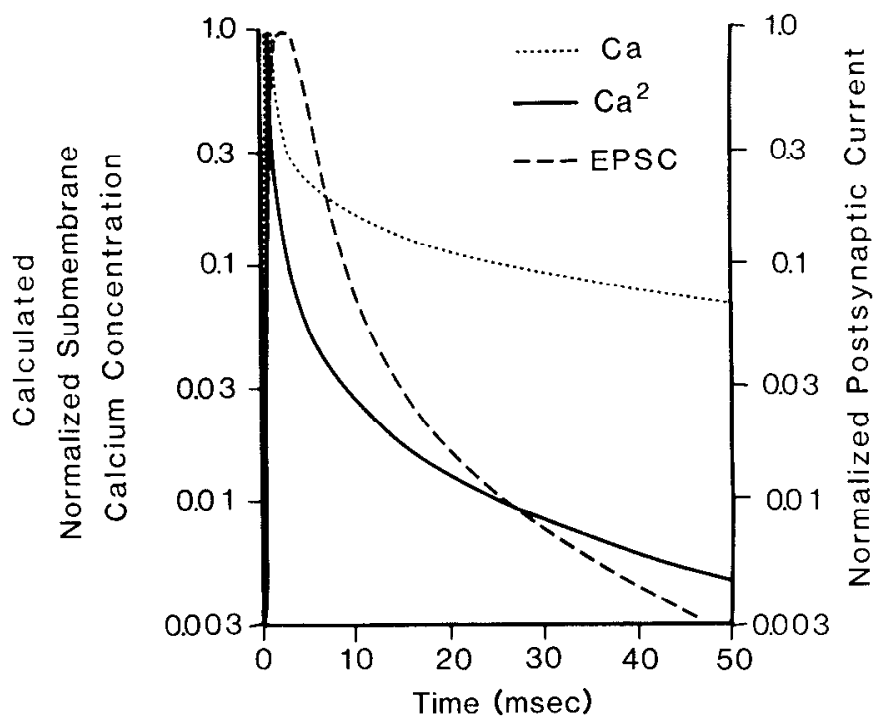

Figure 3. Comparison of predicted time course of transmitter release following a presynaptic action potential to measured time course of postsynaptic current. Logarithmic plots of submembrane calcium concentration (dotted line), and its square (solid line), during and after a single spike beginning at time 0 , normalized to the peak value at the end of the spike. On the left ordinate, 1.0 corresponds to $2.21 \mu \mathrm{M}$ calcium and its square. The dashed line is the normalized excitatory postsynaptic current (EPSC) from a voltage-clamped postsynaptic axon of the squid giant synapse, at $15^{\circ} \mathrm{C}$. On the right ordinate, 1.0 corresponds to $1.64 \mu \mathrm{A}$; adapted from Charlton et al. (1982). The time course of transmitter release will be somewhat faster than this current and should resemble that of the square of the submembrane calcium concentration.

pated after about $10 \mathrm{msec}$ (Fig. $2 B$ ). Figure 3 shows the calcium concentration in the 10 -nm-thick shell just below the plasma membrane as a function of time during and after a nerve impulse. (One synaptic vesicle has a radius of about $25 \mathrm{~nm}$.)

We would like to relate the time course of submembrane calcium following a spike to the time course of transmitter release, but to do so we need a measure of the latter. The time course of transmitter release following an impulse has not been measured directly at the squid giant synapse, owing to the small size of miniature postsynaptic potentials. However, the time course of postsynaptic current following a presynaptic impulse has been measured (Charlton et al., 1982). At neuromuscular junctions, the time course of postsynaptic current is mainly determined by postsynaptic factors, but it is nevertheless only about 3 times slower than the time course of transmitter release (Katz and Miledi, 1965). There is also some evidence that at the squid giant synapse, the turn-off of transmitter release is one of the rate-limiting factors in determining the decay of the postsynaptic current. Transmitter release may be artificially shut off almost instantaneously by suddenly stepping the presynaptic membrane potential to the calcium equilibrium potential after a 1-msec moderate depolarization. When this is done, the postsynaptic current is shortened to less than that following a regular action potential or brief depolarization (R. Llinás, S. Simon, and M. Sugimori, personal communication). Thus, the decline of the postsynaptic current under voltage clamp is probably roughly similar in time course to the decline in transmitter release. The kinetics of the interaction of transmitter with postsynaptic receptors is likely to act as a low-pass filter, slowing somewhat the real time course of transmitter release.

Since transmitter release depends on the square of calcium current during depolarization (Charlton et al., 1982), it seems appropriate to compare the time course of the square of the predicted submembrane calcium concentration to the time course of the release of transmitter, as estimated from the rate of decline of postsynaptic current following a presynaptic action potential. This synaptic current is illustrated in Figure 3. It is, in fact, a little slower than the rate of transmitter release predicted from the square of submembrane calcium, also shown in Figure 3, just as expected from the damping action of postsynaptic kinetics.

We have also used this model to predict the magnitude and time course of synaptic facilitation following a single impulse. We have driven the model with two action potentials separated by various intervals and estimated the peak submembrane calcium concentration reached by the second peak. If the amount of transmitter released by a nerve impulse is related to the square of this calcium concentration, then we may predict synaptic facilitation (expressed as the fractional increase in the synaptic response to the second spike compared to the synaptic response to the first spike) by plotting the fractional increase of the peak squared submembrane calcium concentration reached during the second spike with respect to that reached during the first. This prediction is shown in Figure 4 and is generally similar to two measurements of synaptic facilitation plotted in the same figure.

Due to refractoriness, facilitation may only be measured at intervals greater than about $4 \mathrm{msec}$. This portion of the prediction may be described as the sum of two

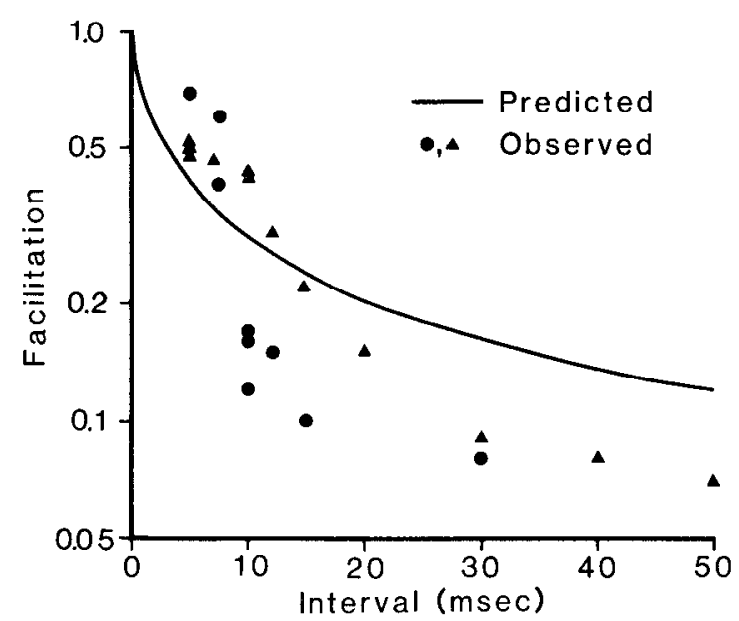

Figure 4. Comparison of predicted and measured synaptic facilitation at the squid giant synapse, plotted on logarithmic coordinates. The line represents predicted facilitation for two spikes separated by the interval shown on the abscissa. Facilitation is the fractional increase of the second postsynaptic potential compared to the first. The symbols $(\boldsymbol{O}, \boldsymbol{\Delta})$ show two examples of experimentally measured synaptic facilitation, at $15^{\circ} \mathrm{C}$; adapted from Charlton and Bittner (1978). 
exponentially declining components. The slowest component has an amplitude of 0.25 and a time constant of $60 \mathrm{msec}$, and the fast component has an amplitude of 0.55 and a time constant of 6 msec. A third, faster component contributes to the early decline of predicted facilitation but is experimentally unmeasurable because it falls in the absolute refractory period. The slow component of facilitation ranges in amplitude (extrapolated to zero time) from 0.18 to 0.25 and in time constant from 22 to $63 \mathrm{msec}$, while the faster component is reported to have an amplitude of $0.9 \pm 0.5$ (SD) and a time constant of $9.5 \pm 2.8$ (SD) msec (Charlton and Bittner, 1978). Thus, the predicted facilitation falls within the reported range of facilitation at this synapse. It is noteworthy that the model predicts both the fast and slow components of facilitation. Evidently, these two components do not necessarily arise from distinct processes, but may merely reflect the second-order removal of calcium from the submembrane space embodied in the diffusion equation.

\section{Discussion}

Our model of intracellular calcium diffusion is only one in a long line of similar models that have been used to simulate intracellular calcium movements in neurons. The first theoretical treatment of this sort was formulated by Blaustein and Hodgkin (1969), who used it to simulate the outward diffusion of intracellular calcium with binding to fixed sites and first-order efflux at the surface, after the axial injection of calcium into the squid giant axon. This model was later modified by Baker et al. (1971) to simulate the inward diffusion of calcium with cytoplasmic binding during and after a prolonged constant influx, in order to estimate the changes in internal calcium distribution caused by a long high frequency train of action potentials. A similar model was developed by Andresen et al. (1979) to compute the intracellular redistribution of calcium released from lipochondria in response to light in a molluscan extraretinal photoreceptor, and included diffusion with cytoplasmic binding and extrusion by a nonsaturable linear surface pump. Smith and Zucker (1980) described another model of intracellular calcium diffusion with cytoplasmic binding and a linear surface pump, which was used to simulate calcium movements during a series of long depolarizing pulses and predict aequorin and arsenazo III responses in Aplysia neurons. Gorman and Thomas (1980) used a model of calcium diffusion with binding to predict the accumulation of submembrane calcium during a prolonged influx. Finally, Llinás et al. (1981b) have considered the rise in intracellular calcium during an action potential due to the diffusion of calcium entering a small cluster of channels in the presynaptic terminal of the squid giant synapse.

In none of these simulations, however, was the distribution of internal calcium following an action potential considered, which is what interested us in the present study. This work represents, therefore, an extension of previous models into a new time domain, and makes a number of new predictions about presynaptic calcium accumulation following an action potential and its expected effects on synaptic transmission.

We have described a model which accounts for the phasic release of transmitter following an action potential, the magnitude and decay of synaptic facilitation, and the rate of disappearance of free calcium from cytoplasm. We tried to formulate the model in the simplest way possible, consistent with current information. It is not likely to be correct in detail. We have already discussed our simplifications regarding uniformly distributed, immobile, and nonsaturable calcium binding, and why we think this is a reasonable representation of axoplasmic calcium buffering. We also used a nonsaturable surface pump to remove cytoplasmic calcium, although our simulations suggest that submembrane calcium reaches levels (Fig. 3 legend) at which the pump may begin to saturate (Requena and Mullins, 1979). However, including reasonable saturation parameters in the model has little effect on its response to two impulses (N. Stockbridge and J. W. Moore, submitted for publication).

We chose to remove calcium from cytoplasm with a surface pump rather than by slow absorption into intracellular organelles such as mitochondria or endoplasmic reticulum. Recent studies of mitochondrial uptake of calcium indicate that this process is very slow at physiological levels of calcium, with a time constant of several minutes (Brinley et al., 1978). Calcium indicator measurements show that internal calcium is extruded much faster than this, suggesting that mitochondria are unlikely to be significant in regulating calcium in the micromolar region. Other considerations of mitochondrial function have led to the same conclusion (Brinley, 1978). The role of endoplasmic reticulum in absorbing small cytoplasmic calcium transients is less clear, since the dynamic properties of this calcium-buffering system are yet to be determined. For this reason, slow uptake into endoplasmic reticulum has not been included explicitly in the present model, although that does not argue against its existence or importance (Blaustein et al., 1978; Henkart et al., 1978; McGraw et al., 1980). We can only say we do not require such a calcium-buffering system, with kinetic properties distinct from rapid cytoplasmic binding, to account for the synaptic properties analyzed here.

We represented the time course of calcium influx during an action potential by a square pulse. This approximation is validated by simulations using a more realistic time course of calcium entry, which shows little difference in the profile of presynaptic calcium concentration following the action potential (J. W. Moore and N. Stockbridge, submitted for publication).

We assumed that voltage-dependent calcium channels are distributed uniformly in the presynaptic membrane. However, calcium entry may be restricted to the synaptic face of the presynaptic terminal (Pumplin et al., 1981). This will increase the density of calcium influx at the synaptic surface, but the absence of calcium entry through a distant nonsynaptic portion of the surface membrane has no bearing on diffusion from the synaptic surface. Calcium actually enters through discrete channels, not uniformly through the whole membrane, but the interchannel equilibration of submembrane calcium occurs in a fraction of a millisecond and does not affect the slower events of concern here (Ilinás et al., 1981b). 
We also make no claim that the particular parameters we have used for influx, binding, extrusion, and resting calcium, and the power-of-two relation between calcium and transmitter release, are necessarily the best ones, just because they predict phasic transmitter release, facilitation, and calcium removal reasonably accurately. We chose these parameters to be within the range of values reported in the literature and we have not attempted to manipulate them in order to provide the best possible fit to any one set of data. For one thing, the time courses of postsynaptic current, synaptic facilitation, and calcium removal as measured with arsenazo are too variable to warrant precise attempts at fitting the data. Moreover, these processes have never all been measured at a single synapse, and such an undertaking would be impractical. Thus, there is no single set of data to which the model could be adjusted. Finally, the postsynaptic current provides only a crude upper limit on the time course of phasic transmitter release. The model could be improved by providing for a presynaptic delay in transmitter release and including the postsynaptic channel kinetics to simulate the postsynaptic response to the release of transmitter, but the required data are not available for this synapse. Thus, the model is too rudimentary to warrant detailed conclusions about the values of particular parameters. Our purpose here was rather to demonstrate that a model of this sort is sufficient to account for the general properties of phasic transmitter release, synaptic facilitation, and arsenazo signals, even though these calcium-dependent processes occur over time scales differing by several orders of magnitude. We feel that, since our model predictions fall within the reported ranges of the behavior of phasic transmitter release (estimated indirectly), synaptic facilitation, and presynaptic calcium concentration changes, the model provides a reasonably good description of the presynaptic processes likely to be controlling these phenomena.

From manipulations of the various parameters in the model, we can discover which components of the system affect which properties of synaptic transmission. Both the phasic release of transmitter and the magnitude and time course of facilitation are influenced most heavily by the rate of diffusion of calcium in cytoplasm, which in turn depends on the proportion of calcium that is bound. These properties of synaptic transmission are naturally also influenced by the relation between submembrane calcium and transmitter release. The rate of calcium extrusion has little effect on these synaptic processes but is the primary determinant of the rate of removal of calcium from cytoplasm. Less obviously, the latter is also quite sensitive to the resting level of calcium, since this sets the basal rate of calcium extrusion and the rate at which small incremental loads are removed. Thus a higher resting level and lower pump rate lead to a similar time course for the removal of calcium entering in an action potential. A more detailed study of the effect of variations in form and level of the various components of this model is presented elsewhere (N. Stockbridge and J. W. Moore, submitted for publication).

Diffusion of calcium accounts for the phasic release of transmitter and the rather rapid decay of facilitation seen at the squid giant synapse. It is surprising that so little attention has been paid to this explanation of these phenomena. It has usually been presumed that calcium is removed from sites of transmitter release by nonlinear processes (Katz and Miledi, 1968) such as active sequestration into presynaptic organelles or extrusion by pumps (Alnaes and Rahamimoff, 1975; Blaustein et al., 1978; Magleby, 1979). Internal diffusion has received only an occasional passing nod as being possibly relevant to presynaptic events following an action potential. Moreover, we know of no attempt in the literature to predict quantitatively the synaptic events that concern us by any model system of presynaptic processes. Therefore, we hope that this study will lead to further refinements in the modeling of presynaptic events and to efforts to test more precisely their predictions.

We believe that the same processes are responsible for the phasic release of transmitter and the fast phases of facilitation at all chemical synapses. An application of this approach to account for phasic release and early facilitation at frog neuromuscular junctions is presented elsewhere (J. W. Moore and N. Stockbridge, submitted for publication). Neither the present simulation nor the squid giant synapse evince prominent prolonged phases of enhanced transmission after presynaptic activity, such as augmentation and potentiation, seen at other synapses (Magleby, 1979; Zengel et al., 1980). These slower synaptic phenomena may reflect the accumulation of calcium in small synaptic terminals with a large surface-tovolume ratio, such that a significant residuum of calcium remains at submembrane sites and elsewhere in cytoplasm even after diffusional equilibration. Under these circumstances, one might expect mechanisms other than diffusion to influence the slow removal of submembrane calcium and lead to augmentation and potentiation. These include the active extrusion of calcium by membrane pumps at the surface or into organelles such as endoplasmic reticulum, and the sodium-dependent release and uptake of calcium by organelles such as mitochondria (Rahamimoff et al., 1980).

\section{References}

Alnaes, E., and R. Rahamimoff (1975) On the role of mitochondria in transmitter release from motor nerve terminals. J. Physiol. (Lond.) 248: 285-306.

Andresen, M. C., A. M. Brown, and S. Yasui (1979) The role of diffusion in the photoresponse of an extraretinal photoreceptor of Aplysia. J. Physiol. (Lond.) 287: 283-301.

Baker, P. F., and W. W. Schlaepfer (1978) Uptake and binding of calcium by axoplasm isolated from giant axons of Loligo and Myxicola. J. Physiol. (Lond.) 276: 103-125.

Baker, P. F., A. L. Hodgkin, and E. B. Ridgway (1971) Depolarization and calcium entry in squid giant axons. J. Physiol. (Lond.) 218: 709-755.

Blaustein, M. P. (1976) The ins and outs of calcium transport in squid axons: Internal and external ion activation of calcium efflux. Fed. Proc. 35: 2574-2578.

Blaustein, M. P., and A. L. Hodgkin (1969) The effect of cyanide on the efflux of calcium from squid axons. J. Physiol. (Lond.) 200: 497-527.

Blaustein, M. P., R. W. Ratzlaff, and E. S. Schweitzer (1978) Calcium buffering in presynaptic nerve terminals. II. Kinetic properties of the nonmitochondrial Ca sequestration mechanism. J. Gen. Physiol. 72: 43-66. 
Brinley, F. J., Jr. (1978) Calcium buffering in squid axons. Annu. Rev. Biophys. Bioeng. 7: 363-392.

Brinley, F. J., Jr., T. Tiffert, A. Scarpa, and L. J. Mullins (1977) Intracellular calcium buffering capacity in isolated squid axons. J. Gen. Physiol. 70: 355-384.

Brinley, F. J., Jr., T. Tiffert, and A. Scarpa (1978) Mitochondria and other calcium buffers of squid axon studied in situ. J. Gen. Physiol. 72: 101-127.

Charlton, M. P., and G. D. Bittner (1978) Facilitation of transmitter release at squid synapses. J. Gen. Physiol. 72: 471-486.

Charlton, M. P., S. J. Smith, and R. S. Zucker (1982) Role of presynaptic calcium ions and channels in synaptic facilitation and depression at the squid giant synapse. J. Physiol. (Lond.) 323: 173-193.

Crank, J., and P. Nicolson (1947) A practical method for numerical evaluation of solutions of partial differential equations of the heat-conduction type. Proc. Cambridge Philos. Soc. 43: 50-67.

DiPolo, R. (1976) The influence of nucleotides on calcium fluxes. Fed. Proc. 35: 2579-2582.

DiPolo, R. (1979) Physiological role of ATP-driven calcium pump in squid axon. Nature 278: 271-273.

DiPolo, R., J. Requena, F. J. Brinley, Jr., L. J. Mullins, A. Scarpa, and T. Tiffert (1976) Ionized calcium concentrations in squid axons. J. Gen. Physiol. 67: 433-467.

Gorman, A. L. F., and M. V. Thomas (1980) Intracellular calcium accumulation during depolarization in a molluscan neurone. J. Physiol. (Lond.) 308: 259-285.

Henkart, M. P., T. S. Reese, and F. J. Brinley, Jr. (1978) Fndoplasmic reticulum sequesters calcium in the squid giant axon. Science 202: 1300-1303.

Hodgkin, A. L., and R. D. Keynes (1957) Movement of labelled calcium in squid giant axons. J. Physiol. (Lond.) 138: 253281.

Katz, B., and R. Miledi (1965) The measurement of synaptic delay, and the time course of acetylcholine release at the neuromuscular junction. Proc. R. Soc. Lond. (Biol.) 161: 483495.

Katz, B., and R. Miledi (1968) The role of calcium in neuromuscular facilitation. J. Physiol. (Lond.) 195: 481-492.

Llinás, R., I. Z. Steinberg, and K. Walton (1981a) Presynaptic calcium currents in squid giant synapse. Biophys. J. 33: 289322.

Llinás, R., I. Z. Steinberg, and K. Walton (1981b) Relation hip between presynaptic calcium current and postsynaptic potential in squid giant synapse. Biophys. J. 33: 323-352.

Magleby, K. L. (1979) Facilitation, augmentation, and potentiation of transmitter release. Prog. Brain Res. 49: 175-182.

McGraw, C. F., A. V. Somlyo, and M. P. Blaustein (1980) Localization of calcium in presynaptic nerve terminals. An ultrastructural and electron microprobe analysis. J. Cell Biol. 85: $228-241$

Miledi, R., and I. Parker (1981) Calcium transients recorded with arsenazo III in the presynaptic terminal of the squid giant synapse. Proc. R. Soc. Lond. (Biol.) 212: 197-211.

Pumplin, D. W., T. S. Reese, and R. Llinás (1981) Are the presynaptic membrane particles the calcium channels? Proc. Natl. Acad. Sci. U. S. A. 78: 7210-7213.

Rahamimoff, R., A. Lev-Tov, and H. Meiri (1980) Primary and secontary regulation of quantal transmitter release: Calcium and sodium. J. Exp. Biol. 89: 5-18.

Requena, J., and L. J. Mullins (1979) Calcium movement in nerve fibres. Q. Rev. Biophys. 12: 371-460.

Smith, S. J., and R. S. Zucker (1980) Aequorin response facilitation and intracellular calcium accumulation in molluscan neurones. J. Physiol. (Lond.) 300: 167-196.

Tillotson, D., and A. L. F. Gorman (1980) Non-uniform $\mathrm{Ca}^{2+}$ buffer distribution in a nerve cell body. Nature 286: 816-817.

Zengel, J. E., K. L. Magleby, J. P. Horn, D. A. McAfee, and P. J. Yarowsky (1980) Facilitation, augmentation, and potentiation of synaptic transmission at the superior cervical ganglion of the rabbit. J. Gen. Physiol. 76: 213-231. 